\title{
Dynamic Comprehensive Evaluation of Ecological Environment of 12 Provinces and Cities in Western China
}

\author{
Hongmei Zhang ${ }^{1,2}$, Teng $\mathrm{Li}^{1,2}$, Yazhong Chen ${ }^{1,2}$ \\ ${ }^{1}$ Guizhou Science and Technology Innovation and Venture Capital Research Institute, \\ Guizhou University of Finance and Economics, Guiyang 550025, China \\ ${ }^{2}$ School of Finance, Guizhou University of Finance and Economics, Guiyang 550025, China
}

\section{我国西部 12 省市生态环境的动态综合评价}

\author{
张红梅 $^{1,2}$, 李腾 ${ }^{1,2}$, 陈亚中 ${ }^{1,2}$ \\ ${ }^{1}$ 贵州财经大学, 贵州科技创新创业投资研究院, 贵阳 550025, 中国 \\ 2 贵州财经大学, 金融学院, 贵阳 550025, 中国
}

\begin{abstract}
The problem of ecological environment is a global problem. It is a basic material condition for human beings to survive and engage in various production practices, and has a tremendous impact on our economic and social life and development. In recent years, the overall situation of the ecological environment in western China has been severe. On the one hand, it is because of the inherent fragility of the ecological environment in the western region. On the other hand, it is due to human, economic, and political and legal factors. Therefore, the western region is faced with the dual task of protecting the environment and developing the economy. In the face of these dual tasks, the western region must choose a development model based on the coordinated development of the environment and economy. Therefore, this
\end{abstract}

基金项目: 本文由贵州省科技计划项目黔科合基础 [2016] 1534-4 号资助。项目名称: 贵州新型城镇化金融 支持效率研究。

作者简介: 张红梅, 教授、硕士生导师, 任职于贵州财 经大学金融学院, 研究方向: 金融与区域发展、贫困地 区金融。Emai1：zhm1035@qq. com。李腾、陈亚中, 男, 贵州财经大学金融学院 2014 级研究生硕士。 paper selects data from 12 provinces and cities in the western part of China for 6 years, adopts multiple evaluation methods to conduct a dynamic comprehensive assessment of the western ecological environment, and provides some corresponding countermeasures for the coordinated development of the environment and economy in the western ecologically fragile region and suggest.

Keywords: western ecologically fragile area; ecological environment; dynamic comprehensive evaluation

\section{摘要}

生态环境问题是一个全球所要面临的问 题, 它是人类赖于生存和从事各种生产实践 的基本物资条件, 对我们经济社会的生活和 发展有着巨大的影响。近年来, 我国西部的生 态环境整体形势严峻, 一方面是因为西部地区 生态环境固有的脆弱性, 另一方面是因为人为、 经济和政治法律因素。因此, 西部地区面临着 保护环境和发展经济的双重任务。在这些双 重任务面前, 西部地区必须要选择基于环境与 经济协调发展的的开发模式。因此, 本文选 取了我国西部地区 12 个省市 6 年的数据, 
采用多种评价方法对西部生态环境进行动态 综合评价, 同时为西部生态脆弱区的环境与 经济协调发展提供了一些相应的对策和建议。 关键词: 西部生态脆弱区; 生态环境; 动态 综合评价

\section{1 引言}

近年来我国经济迅速发展, 西部的经济水平 也是大幅度提高, 但是西部经济发展的过程 中对环境的索取与破坏也是过度和严重的。 西部生态环境本来就很脆弱, 因此在发展的 过程中很有必要对西部现有的发展水平进行 综合评价研究, 以此找到发展中的问题, 找 到一条更有效率更合理的发展之路。

目前, 国内外学者对生态环境进行评价 都取得了一定的成果。Matthew 和 Luck (2001) 将人类生态足迹模型和生态系统过 程模型相结合, 得到影响生态系统发展的重 要因素, 把改进的生态足迹法应用到城市生 态系统评价中去, 并对美国的主要 20 个城 市的生态系统进行了评价 [1]。李玉平 (2007) 以自然一经济一社会概念框架模型。从土地 自然生态安全、土地社会生态安全和土地经 济生态安全三个方面来构建指标体系。张华 （2008）在《西部地区生态环境综合评价研 究》一文中, 联系西部各省区的具体实际, 在参考中国科学院可持续发展研究组建立的 环境支持系统指标体系的基础上, 构建了西 部生态环境评价指标体系 [2]。陈圆圆（2010） 从土地生态系统压力、土地生态系统状态、 土地生态系统响应三个方面来构建评价指标 体系, 充分考虑自然、经济、社会等诸多因 素, 但评价精度要受到指标代表性及其权重 合理性的影响。董晓晓 (2014) 在《西部生 态脆弱区土地生态状况评价及预测》一文中, 以西部生态脆弱区甘肃省榆中县为研究区域, 利用 GIS 对研究区的土地利用变化、地形地 貌、植被覆盖、景观格局和土壤侵蚀进行了 分析, 反映了单因素影响下的土地生态风险 状况。而本文主要是建立了一套评价生态环 境的适当指标集, 用多种方法对西部生态脆 弱区进行动态综合评价, 根据研究结果对西 部 12 个省市进行综合排序, 并提出相应的政 策建议。

\section{2 多种方法动态模型简介}

在对我国西部生态环境进行动态综合评 价, 主要采用了拉开档次法、理想点法和摘 值法三种方法相结合。我们将这三种评价方 法有机地结合应用到对西部生态环境的评价 中去, 能够很好地体现出我们是用运动的观 点、发展的观点和客观的观点去看待和处理 变化的事物。

\section{1 拉开档次法的基本原理}

拉开档次法主要是针对由时序的立体数 据表的综合评价问题的特殊性, 提出来的一 种新的确定权重系数的方法。它多应用于经 济管理、干部考核等多指标决策问题。

假设有 $\mathrm{m}$ 个评价对象 $\mathrm{G}_{1}, \mathrm{G}_{2}, \cdots, \mathrm{G}_{\mathrm{m}}$ 有 $\mathrm{n}$ 个评价指标 $\mathrm{x}_{1}, \mathrm{x}_{2}, \cdots, \mathrm{x}_{\mathrm{n}}$, 且由时间 $\mathrm{t}_{1}$, $\mathrm{t}_{2}, \cdots, \mathrm{t}_{\mathrm{r}}$ 顺序排列的原始数据 $\left\{\mathrm{x}_{\mathrm{ij}}\left(\mathrm{t}_{\mathrm{k}}\right)\right\}$, $\left\{\mathrm{x}_{\mathrm{ij}}\left(\mathrm{t}_{\mathrm{k}}\right)\right\}$ 组成了一个时序立体数据表.

定义: 由时序立体数据表支持的综合评 价问题, 称为动态综合评价问题般可表示为: $y_{i}\left(t_{k}\right)=F\left\{\begin{array}{c}w_{1}\left(t_{k}\right), w_{2}\left(t_{k}\right), \ldots, w_{m}\left(t_{k}\right) ; \\ x_{1}\left(t_{k}\right), x_{2}\left(t_{k}\right), \ldots, x_{n}\left(t_{k}\right)\end{array}\right\}$
$i=1,2, \ldots, n ; k=1,2, \ldots, r$
其中 $y_{i}\left(t_{k}\right)$ 表示评价对象 $G_{i}$ 在时刻 $t_{k}$ 时 的综合评价值, $F$ 表示函数解析式, $\mathrm{w}_{\mathrm{i}}\left(\mathrm{t}_{\mathrm{k}}\right)$ 表示评价指标 $\mathrm{x}_{\mathrm{i}}$ 在时刻 $\mathrm{t}_{\mathrm{k}}$ 时的权重系数。

拉开档次法就是取综合评价函数

$$
\begin{gathered}
y_{i}=w_{1} x_{i 1}+w_{2} x_{i 2}+\cdots+w_{n} x_{i n} \\
i=1,2, \ldots, n
\end{gathered}
$$

要确定 $\mathrm{w}_{\mathrm{i}}$ 的值就是要使得评价对象 $\mathrm{G}_{1}$, $\mathrm{G}_{2}, \ldots, \mathrm{G}_{\mathrm{m}}$ 之间的差异尽可能地大, 评价 对象之间的差异可以由 $\sigma^{2}=\sum_{1}^{\mathrm{m}}\left(\mathrm{y}_{\mathrm{i}}-\overline{\mathrm{y}}\right)^{2}$ 来 表示。将原始数据标准化, 可得到 $\overline{\mathrm{y}}=\frac{\sum \mathrm{y}_{\mathrm{i}}}{\mathrm{m}}=0$, 此时有 $\sigma^{2}=\sum \mathrm{y}_{\mathrm{i}}{ }^{2}=\mathrm{Y}^{\mathrm{T}} \mathrm{Y}=$ $(W X)^{\mathrm{T}} \mathrm{XW}=\mathrm{W}^{\mathrm{T}} \mathrm{HW}$, 其中,

$$
\begin{aligned}
& Y=\left(\begin{array}{c}
\mathrm{y}_{1} \\
\mathrm{y}_{2} \\
\vdots \\
\mathrm{y}_{\mathrm{m}}
\end{array}\right), \quad W=\left(\begin{array}{c}
\mathrm{w}_{1} \\
\mathrm{w}_{2} \\
\vdots \\
\mathrm{w}_{\mathrm{m}}
\end{array}\right), H=\mathrm{X}^{\mathrm{T}} \mathrm{X}, \\
& X=\left(\begin{array}{ccc}
\mathrm{x}_{11} & \cdots & \mathrm{x}_{1 \mathrm{n}} \\
\vdots & \ddots & \vdots \\
\mathrm{x}_{\mathrm{m} 1} & \cdots & \mathrm{x}_{\mathrm{mn}}
\end{array}\right) \mathrm{t}_{\mathrm{k}}
\end{aligned}
$$

当限制 $\|W\|=1$, 即 $w_{1}{ }^{2}+w_{2}{ }^{2}+\cdots+$ $\mathrm{w}_{\mathrm{n}}{ }^{2}=1$ 时取为 $W$ 对称阵 $H$ 的最大特征值 
$\lambda_{\text {max }}(H)$ 所对应的特征向量, 则 $\sigma^{2}$ 取值最大, 且此时 $\max \left\{\sigma^{2}\right\} \lambda_{\text {max }}(H)$ 分别对时刻 $\mathrm{t}_{\mathrm{k}}$ 处的截 面 数 据 $\left\{\mathrm{x}_{\mathrm{ij}}\left(\mathrm{t}_{\mathrm{k}}\right)\right\}(\mathrm{i}=1,2, \ldots, \mathrm{m} ; \mathrm{j}=$ $1,2, \ldots, n)$ 应用拉开档次法, 可以求得 该时刻与指标 $\mathrm{x}_{\mathrm{i}}(\mathrm{i}=1,2, \ldots, \mathrm{n})$ 相对应的 权重 系 数 $w_{i}(i=1,2, \ldots, n ; j=$ $1,2, \ldots, r)$, 之后再代入综合评价函数 $y_{i}=w_{1} x_{i 1}+w_{2} x_{i 2}+\cdots+w_{n} x_{i n}$ 可得综合得分。

\section{2 摘值法的基本原理}

在得到评价对象 $\mathrm{G}_{\mathrm{i}}$ 在时刻 $\mathrm{t}_{\mathrm{k}}$ 时的综合评 价值 $\mathrm{y}_{\mathrm{i}}\left(\mathrm{t}_{\mathrm{k}}\right)(\mathrm{i}=1,2, \ldots, \mathrm{m} ; \mathrm{k}=$ $1,2, \ldots, r$ 之后, 由于对不同时刻的重视程 度不同, 得到最后评价结果也不同, 因此要 想得到合理的评价结果, 科学的确定时间权 向量 $v=\left(v_{1}, v_{2}, \ldots, v_{\mathrm{r}}\right)^{\mathrm{T}}$ 是关键。时间权 向量、就是表明对不同时刻的重视程度的, 可以根据不同的准则, 应用不同的主观或客 观赋权方法来确定。

下面介绍熵值法 [7] 确定时间权向量 $v$ :

先定义熵 $\mathrm{I}=-\sum_{1}^{\mathrm{r}} v_{\mathrm{k}} \ln v_{\mathrm{k}}$

熵为热力学中的一个名词, 在信息论中 又被称为是平均信息量, 即它是信息的一个 度量。嫡值越大说明它所含的信息量越小, 与此同时时间权向量的熵也反映了对样本的 集结过程中权重包含信息的程度。

再定义 “时间度” $\lambda=\sum_{1}^{\mathrm{r}} \frac{\mathrm{r}-\mathrm{k}}{\mathrm{r}-1} v_{\mathrm{k}}$, 特别当 $v=(1,0, \ldots, 0), \lambda=1$;

当 $v=(0,0, \ldots, 1)$ 时, $\lambda=0$; 当 $v=\left(\frac{1}{\mathrm{r}}, \frac{1}{\mathrm{r}}, \ldots, \frac{1}{\mathrm{r}}\right)$ 时, $\lambda=0.5$

“时间度” $\lambda$ 的大小体现的是集结过程中 对时序的重视程度, 如表 2-3 所示:

\begin{tabular}{|c|c|}
\multicolumn{2}{|c|}{ 表 $2-1$ “时间度” $\lambda$ 的标度参数表 } \\
\hline 赋值 & 说明 \\
\hline 0.1 & 非常不重视近期数据 \\
\hline 0.3 & 较不重视近期数据 \\
\hline 0.5 & 同样重视所用时期数据 \\
\hline 0.7 & 较重视近期数据 \\
\hline 0.9 & 非常重视近期数据 \\
\hline $0.2,4,0.6$, & 对应以上两相邻判断的 \\
0.8 & 中间情况 \\
\hline
\end{tabular}

从表 2-1 可看出 $\lambda$ 的值越大, 反映了评价
者对距评价时刻 $\mathrm{t}_{\mathrm{r}}$ 较近期额数据的重视程度 越小, 对距评价时刻 $\mathrm{t}_{\mathrm{r}}$ 较远期额数据的重视 程度越大。 $\lambda$ 接近 0 时距评价时刻 $\mathrm{t}_{\mathrm{r}}$ 较远期额 数据几乎不起作用, 这种情况主要用于已经 发生的完成时态的动态综合评价问题中; $\lambda$ 接 近 1 时距评价时刻 $\mathrm{t}_{\mathrm{r}}$ 较近期额数据几乎不起 作用, 这种 J 隋况主要用于带有预测性质的 将来时态的动态综合评价问题; 当 $\lambda=0.5$ 时 反映了评价者对对各个时段的重视程度一样, 没有偏向任何一方。

最后介绍确定准则: 在已经给定 “时间 度” 的条件下, 为了找出适合样本集结的时 间权向量, 我们以尽可能多地挖掘样本的信 息并兼顾被评价对象在时序上的差异信息为 标准, 用数学语言来描述此准则, 即解如下 非线性规划问题:

$$
\left\{\begin{array}{c}
\max \left(-\sum_{1}^{\mathrm{r}} v_{\mathrm{k}} \ln v_{\mathrm{k}}\right) \\
\text { s.t. } \lambda=\sum_{1}^{\mathrm{r}-\mathrm{r}-\mathrm{k}} v_{\mathrm{k}} \\
\sum_{1}^{\mathrm{r}} v_{\mathrm{k}}=1, \quad v_{\mathrm{k}} \in\left[\begin{array}{ll}
0, & 1
\end{array}\right] \\
\mathrm{k}=1,2, \quad \ldots, \mathrm{r}
\end{array}\right.
$$

\section{3 理想点法的基本原理}

各个时间点上各评价对象的静态综合评 价值, 设这些评价值的时间序列构成的矩阵 如下:

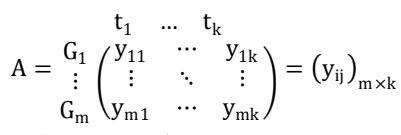

其中 $y_{i j}$ 表示评价对象 $G_{i}(i=1,2, \ldots, m)$ 在 时间点 $t_{j}(j=1,2, \ldots, r)$ 上的静态综合评 价值。

$$
b_{i j}\left\{\begin{array}{c}
\text { 设增长矩阵为 } B=\left(b_{i j}\right)_{m \times r}, \text { 其中 } \\
0, i=1,2, \ldots, m ; j=1 \\
\frac{\left(y_{i j}-y_{i(j-1)}\right)}{\left|y_{i(j-1)}\right|}, i=1,2, \ldots, m ; j=1,2, \ldots, r
\end{array}\right.
$$

则 $\mathrm{b}_{\mathrm{ij}}$ 表示评价对象 $\mathrm{G}_{\mathrm{i}}$ 的综合评价值从时间点 $\mathrm{t}_{(\mathrm{j}-1)}$ 到 $\mathrm{t}_{\mathrm{j}}$ 的增长变化的情况, 另外为了保证 $b_{i j}$ 的增减变化情况同这里给分母加上了绝对 值, $\mathrm{b}_{\mathrm{i} 1}=0$ 表示在基期 $\mathrm{t}_{1}$ 评价值的增长变化 为零。静态综合评价值的变化方向一致, 把 静态综合评价矩阵 $\mathrm{A}$ 和增长变化矩阵 $\mathrm{B}$ 加权 合成就能得到要求的动态综合评价矩阵 $\mathrm{C}$ 。

$$
\mathrm{C}=\left(\mathrm{c}_{\mathrm{ij}}\right)_{\mathrm{m} \times \mathrm{r}}, \quad \mathrm{c}_{\mathrm{ij}}=\alpha \mathrm{y}_{\mathrm{ij}}+\beta \mathrm{b}_{\mathrm{ij}}, \quad \alpha+\beta=1
$$


其中 $\alpha 、 \beta$ 表示的是静态评价值和增长变化值 的相对重要程度, 当 $\alpha=0, \beta=1$ 时评价过 程中只考虑了增长变化程度, 当 $\alpha=1$, $\beta=0$ 时评价过程中只考虑了静态评价值情况, 其余情况则在评价过程中两者兼顾。所以由 $\alpha$ 、 $\beta$ 的取值不同可以更充分的考虑到评价对象静 态评价值和动态增长变化两方面的内容。

然后我们对矩阵 c 用理想点法, 先构造 理想时间序列和负理想序列:

$$
\begin{aligned}
\mathrm{c}^{+}=\mathrm{c}_{1}^{+}, \mathrm{c}_{2}^{+}, \ldots, \mathrm{c}_{\mathrm{r}}^{+}, \mathrm{c}^{-} \\
\\
=\mathrm{c}_{1}^{-}, \mathrm{c}_{2}^{-}, \ldots, \mathrm{c}_{\mathrm{r}}^{-}
\end{aligned}
$$

其中

$\mathrm{c}_{\mathrm{k}}^{+}=\max \left\{\mathrm{c}_{\mathrm{ij}} \mid \mathrm{j}=1,2, \ldots, \mathrm{m}\right\}, \mathrm{k}=$

$1,2, \ldots, r$

$$
\begin{aligned}
c_{k}^{-}=\max \left\{c_{i j} \mid j\right. & =1,2, \ldots, m\}, k \\
& =1,2, \ldots, r
\end{aligned}
$$

根据理想点的算法, 评价对象 $\mathrm{G}_{\mathrm{k}}$ 在时间 点 $\mathrm{t}_{\mathrm{j}}$ 上的动态综合评价值 $\mathrm{c}_{\mathrm{ij}}$ 到 $\mathrm{c}^{+}, \mathrm{c}^{-}$的距离分 别为:

$$
\begin{array}{r}
d_{j}^{+}=\left[\sum_{1}^{r} v_{j}\left(c_{k j}-c_{j}^{+}\right)^{2}\right]^{1 / 2}, j \\
=1,2, \ldots, m \\
d_{j}^{-}=\left[\sum_{1}^{r} v_{j}\left(c_{k j}-c_{j}^{-}\right)^{2}\right]^{1 / 2}, j \\
=1,2, \ldots, m
\end{array}
$$

其中 $v_{\mathrm{j}}$ 表示时间点 $\mathrm{t}_{\mathrm{j}}$ 的权重, 则第 $\mathrm{k}$ 个评 价对象与理想解得相对接近程度为:

$$
\mathrm{s}_{\mathrm{k}}=\frac{\mathrm{d}_{\mathrm{k}}^{-}}{\left(\mathrm{d}_{\mathrm{k}}^{+}+\mathrm{d}_{\mathrm{k}}^{-}\right)}, \mathrm{k}=1,2, \ldots, \mathrm{m}
$$

最后把 $\mathrm{s}_{\mathrm{k}}$ 作为评价对象 $\mathrm{G}_{\mathrm{k}}$ 的最终动态综 合评价值, 这里 $s_{\mathrm{k}} \in[0,1]$ 。如果 $s_{\mathrm{k}}$ 的值越 大, 则评价对象 $\mathrm{G}_{\mathrm{k}}$ 越接近理想点并远离负理 想点, 此时动态综合评价值就越高, 排名就 越靠前; 反之, 评价对象 $\mathrm{G}_{\mathrm{k}}$ 越接近负理想点 并远离理想点, 此时动态综合评价值就越低, 排名就越靠后。

\section{3 实证分析}

\section{1 构建指标体系}

本文在借鉴了王金叶、程道品、胡新添、 李铭、曹连海、郝仕龙、陈南祥 $[5][6]$ 等人

\begin{tabular}{|c|c|c|c|}
\hline & & 指标名称 (单位) & 指标 \\
\hline \multirow{15}{*}{$\begin{array}{l}\text { 西 } \\
\text { 部 } \\
\text { 生 } \\
\text { 态 } \\
\text { 脆 } \\
\text { 弱 } \\
\text { 区 } \\
\text { 综 } \\
\text { 合 } \\
\text { 评 } \\
\text { - }\end{array}$} & \multirow{5}{*}{$\begin{array}{l}\text { 资 } \\
\text { 源 }\end{array}$} & 森林覆盖率（\%） & $\mathrm{X} 1$ \\
\hline & & 自然保护区占辖区面积比重 (\%) & $\mathrm{X} 2$ \\
\hline & & 水土流失治理面积（千公顷） & $\mathrm{X} 3$ \\
\hline & & 造林面积（千公顷） & $\mathrm{X} 4$ \\
\hline & & 人工湿地面积 (千公顷) & $\mathrm{X} 5$ \\
\hline & \multirow{3}{*}{$\begin{array}{l}\text { 环 } \\
\text { 境 }\end{array}$} & 废水排放总量（万吨） & $\mathrm{X} 6$ \\
\hline & & 二氧化硫排放量（吨） & $\mathrm{X} 7$ \\
\hline & & 工业污染治理完成投资（万元） & $\mathrm{X} 8$ \\
\hline & \multirow{3}{*}{$\begin{array}{l}\text { 经 } \\
\text { 济 }\end{array}$} & 农林牧渔业总产值（万元） & $\times 9$ \\
\hline & & $\begin{array}{c}\text { 城镇居民家庭人均可支配收入 } \\
\text { (元) }\end{array}$ & $\mathrm{X} 10$ \\
\hline & & 人均地区生产总产值（元/人） & $\mathrm{X} 11$ \\
\hline & \multirow{4}{*}{$\begin{array}{l}\text { 社 } \\
\text { 会 }\end{array}$} & 总人口数 $($ 万人) & $\mathrm{X} 12$ \\
\hline & & 居民消费水平（元） & $\mathrm{X} 13$ \\
\hline & & 人口自然增长率 $(\%)$ & $\mathrm{X} 14$ \\
\hline & & 人均水资源量（立方米/人） & $\mathrm{X} 15$ \\
\hline
\end{tabular}
的基础上, 结合区域生态评价相关理论和指
标选取原则, 构建了一套西部生态环境脆弱 综合评价指标体系。该体系主要包括资源、 环境、经济和社会四个子系统, 共 15 个指标, 如表 3-1 所示。选取了我国西部 12 个省市时 间段为 2012-2017 共六年的数据, 所收集的 数据全部来源于 2012-2017 年我国统计年鉴。

\section{表 3-1 我国西部生态脆弱区综合评价指标体系}

\section{2 我国西部生态脆弱区综合评价}

\subsection{1 基于拉开档次法的各个指标在不同时刻 权重的确定}

利用拉开档次法来确定各个指标在不同 时刻的权重系数的思路是：首先将我国西部 12 个省市从 2012-2017 年各个指标的原始数 据进行指标类型一致化、无量纲化处理。然 后根据拉开档次法的原理, 得到指标在不同 时刻的权重, 本文借助 SPSS20.0 统计软件来 进行数据标准化处理, 然后再通过计算机计 算出各个指标的权重, 并对得到的各个权重 系数情况, 分析在 6 年的环境发展中, 对环 境影响比较大的指标, 从而为有关部门要提 高环境质量提供一些措施及政策性的建议。 本文所采用的原始数据, 全部来源于各年统 计年鉴。

设定: $\mathrm{T}=6, \mathrm{~m}=12, \mathrm{n}=18$, 首先, 对原始数据 $\left\{x_{i j}\left(t_{k}\right)\right\}$ 进行指标类型一致化、 无量纲化, 然后再计算出对称矩阵 $H_{k}=\left(X_{K}\right)^{T} X_{K}(K=1,2,3,4,5,6)$ 
根据之前的理论, 计算出与 $\mathrm{H}_{\mathrm{K}}$ 相对应的 最大特征值 $\lambda_{\text {max }}(\mathrm{k})$ 及其对应的特征向量即 各个指标在不同时刻的权重系数向量

所以, 各个指标在不同时刻的权重如表 3-2 所示。

表 3-2 指标在不同时刻的权重系数

\begin{tabular}{|c|c|c|c|c|c|c|}
\hline 指标 & 2012 & 2013 & 2014 & 2015 & 2016 & 2017 \\
\hline $\mathrm{X}_{1}$ & 0.2578 & 0.2585 & 0.2696 & 0.2407 & 0.2505 & 0.2185 \\
\hline $\mathrm{X}_{2}$ & 0.2494 & 0.2636 & 0.2651 & 0.2516 & 0.2694 & 0.2616 \\
\hline $\mathrm{X}_{3}$ & 0.2466 & 0.2648 & 0.2683 & 0.2770 & 0.2851 & 0.2849 \\
\hline $\mathrm{X}_{4}$ & 0.2703 & 0.2678 & 0.2808 & 0.2641 & 0.2439 & 0.2701 \\
\hline $\mathrm{X}_{5}$ & 0.1014 & 0.1089 & 0.1032 & 0.0868 & 0.0941 & 0.1273 \\
\hline $\mathrm{X}_{6}$ & 0.2363 & 0.2282 & 0.2299 & 0.2176 & 0.2320 & 0.2124 \\
\hline $\mathrm{X}_{7}$ & 0.3268 & 0.3228 & 0.3178 & 0.3072 & 0.3053 & 0.3132 \\
\hline $\mathrm{X}_{8}$ & 0.3467 & 0.2826 & 0.2275 & 0.3072 & 0.2561 & 0.2887 \\
\hline $\mathrm{X}_{9}$ & 0.3012 & 0.2923 & 0.2888 & 0.2693 & 0.2740 & 0.2650 \\
\hline $\mathrm{X}_{10}$ & 0.2210 & 0.2301 & 0.2489 & 0.2725 & 0.2835 & 0.2835 \\
\hline $\mathrm{X}_{11}$ & 0.1787 & 0.1988 & 0.1993 & 0.2108 & 0.2059 & 0.2270 \\
\hline $\mathrm{X}_{12}$ & 0.3100 & 0.2935 & 0.2963 & 0.2704 & 0.2753 & 0.2532 \\
\hline $\mathrm{X}_{13}$ & 0.1853 & 0.2405 & 0.2561 & 0.2676 & 0.2601 & 0.2688 \\
\hline $\mathrm{X}_{14}$ & 0.2966 & 0.2995 & 0.3012 & 0.3061 & 0.3077 & 0.2971 \\
\hline $\mathrm{X}_{15}$ & 0.2333 & 0.2495 & 0.2465 & 0.2428 & 0.2580 & 0.2455 \\
\hline
\end{tabular}

从表 3-2 中可以看出这 15 个指标的重要 趋势, 按其重要程度的大小可以分为四类: 第一类是工业治理完成投资、二氧化硫排放 量、农林牧渔业总产值、总人口数这些指标 所占的比重最大; 第二类是人口自然增长率、 造林面积、森林覆盖率、自然保护区占辖区 面积比重、水土流失面积的重要性相对次之; 第三类是废水排放总量、人均水资源量、城 镇居民家庭人均可支配收入; 第四类是居民 消费水平、人均地区生产总值、人工湿地面 积。这样可以看出工业污染及其治理情况以 及造林面积等生态因素与人口的发展变化对 西部综合发展情况的影响最大。

\subsection{2 我国西部 12 个省市的综合得分}

为了突出各项指标在不同时刻的作用, 本文采用线性加权法即:

$$
\begin{gathered}
\mathrm{y}_{\mathrm{i}}\left(\mathrm{t}_{\mathrm{k}}\right)=\mathrm{w}_{1}\left(\mathrm{t}_{\mathrm{k}}\right) \mathrm{x}_{\mathrm{i} 1}\left(\mathrm{t}_{\mathrm{k}}\right)+\mathrm{w}_{2}\left(\mathrm{t}_{\mathrm{k}}\right) \mathrm{x}_{\mathrm{i} 2}\left(\mathrm{t}_{\mathrm{k}}\right)+\cdots \\
+\mathrm{w}_{\mathrm{n}}\left(\mathrm{t}_{\mathrm{k}}\right) \mathrm{x}_{\mathrm{in}}\left(\mathrm{t}_{\mathrm{k}}\right)
\end{gathered}
$$

$\mathrm{K}=1 ， 2 ， 3 ， 4 ， 5 ， 6 ; \mathrm{i}=1,2 ， \ldots, \mathrm{m}$

其中 $\mathrm{y}_{\mathrm{i}}\left(\mathrm{t}_{\mathrm{k}}\right)$ 表示第 $\mathrm{i}$ 个评价对象在 $\mathrm{t}_{\mathrm{k}}$ 时刻 处的综合得分值, $w_{i}\left(t_{k}\right)$ 表示 $X_{i}$, 指标在 $t_{k}$ 时 刻处的权重。

得到西部 12 个省市在不同年份的综合得 分值, 如表 3-3 所示:
表 3-3 西部 12 省市 2008-2013 年的综合得

$$
\text { 分值 } \mathrm{y}_{\mathrm{i}}\left(\mathrm{t}_{\mathrm{k}}\right)
$$

\begin{tabular}{|l|l|l|l|l|l|l|}
\hline 省市 & 2012 & 2013 & 2014 & 2015 & 2016 & 2017 \\
\hline 新疆 & 0.6399 & 0.4272 & 0.5860 & 0.5002 & 0.3806 & 0.2280 \\
\hline 甘肃 & 0.9790 & 0.9217 & 1.0434 & 1.0837 & 0.7820 & 1.4441 \\
\hline 青海 & 2.2025 & 1.9871 & 1.9800 & 1.9063 & 1.9298 & 1.8497 \\
\hline 内蒙古 & 2.4996 & 2.5714 & 2.3273 & 3.0918 & 2.6406 & 3.4214 \\
\hline 陕西 & 0.2462 & 0.9302 & 1.1598 & 1.0565 & 1.1213 & 0.7548 \\
\hline 宁夏 & 1.4908 & 1.5750 & 1.5948 & 1.5702 & 1.5076 & 1.5060 \\
\hline 四川 & 2.7635 & 2.1274 & 2.0897 & 2.0683 & 1.7431 & 1.5521 \\
\hline 贵州 & 0.7284 & 0.9201 & 0.9652 & 0.7891 & 0.8588 & 0.5017 \\
\hline 云南 & 0.2890 & 0.3893 & 0.6080 & 0.9529 & 1.0651 & 1.0101 \\
\hline 重庆 & 0.1221 & 0.1641 & 0.0227 & 0.1418 & 0.1203 & 0.0096 \\
\hline 广西 & 1.7542 & 1.6045 & 1.5333 & 0.7069 & 0.7875 & 0.6979 \\
\hline 西藏 & 1.6339 & 1.6277 & 1.5715 & 1.8852 & 1.7785 & 1.8972 \\
\hline
\end{tabular}

\subsection{3 摘值法确定时间权向量}

在利用熵值法确定时间权重 $v_{\mathrm{k}}$ 时, 我们 事先要经过有关专家的意见确定 “时间度” $\lambda$ 的值, 在这里我们取 $\lambda=0.1, r=6$, 我们就用线性规划模型:

$$
\left\{\begin{array}{c}
\max \left(-\sum_{1}^{\mathrm{r}} v_{\mathrm{k}} \ln v_{\mathrm{k}}\right) \\
\text { s.t. } \lambda=\sum_{1}^{\mathrm{r}} \frac{\mathrm{r}-\mathrm{k}}{\mathrm{r}-1} v_{\mathrm{k}} \\
\sum_{1}^{\mathrm{r}} v_{\mathrm{k}}=1, \quad v_{\mathrm{k}} \in\left[\begin{array}{ll}
0,1 \\
\mathrm{k}=1,2, \quad \ldots, 6
\end{array}\right.
\end{array}\right.
$$

利用 Lingo 解线性规划以上模型 , 得到 时间权向量 $v_{\mathrm{k}}=(0.0029,0.0086,0.0255$, $0.0755,0.2238,0.6637)$

\subsection{4 我国西部 12 省市生态环境的动态综合 评价结果}

根据之前的理论, 我们知道由综合评价 值 (综合得分值) 构成的静态评价矩阵 A, 同时根据增长矩阵计算公式得到增长矩阵 $\mathrm{B}$ 。 在这里, 我们认为静态评价值和增长变化值 的重要性是相等的, 所以 $\alpha=\beta=0.5$, 代 入公式 $C=\left(c_{i j}\right)_{m \times r}, c_{i j}=\alpha \cdot y_{i j}+\beta \cdot b_{i j}, \alpha+$ $\beta=1$, 得到矩阵 $C$

由矩阵 C 可以得到正理想时间序列和 负理想时间序列: $\mathrm{C}+=(1.3817,1.8542 ， 1.1162 ， 2.6914 ，$ $1.2473,1.8585)$ C-=(0.0610, 0.0473, $-0.4194,0.0839$, $-0.0156,-0.4553$ )

再由评价对象 $\mathrm{G}_{\mathrm{K}}$ 在时间点 $\mathrm{t}_{\mathrm{j}}$ 上的动态综 合评价值 $\mathrm{c}_{\mathrm{kj}}$ 到 $\mathrm{C}^{+}, \mathrm{C}^{-}$的距离公式, 即: $\mathrm{d}^{+}=(1.8275,0.9637,0.9287,0.2746$, $1.5061,1.0772,1.0585,1.6669,1.2928$, 
$1.9994,1.5161,0.8729)$

$\mathrm{d}^{-}=(0.3367,1.3273,1.2488,2.0464$,

$0.6724,1.0839,1.0948,0.3778,0.8504$,

$0.7167,0.6784,1.2865$ )

由第 $\mathrm{k}$ 个评价对象与理想解得相对接近程度 公式, 即

$$
\mathrm{s}_{\mathrm{k}}=\frac{\mathrm{d}_{\mathrm{k}}^{-}}{\left(\mathrm{d}_{\mathrm{k}}^{+}+\mathrm{d}_{\mathrm{k}}^{-}\right)}, \mathrm{k}=1,2, \ldots, \mathrm{m}
$$

最后, 得到我国西部 12 个省市的综合排序, 如表 3-4 所示:

表 3-4 西部 12 省市的综合评价排序

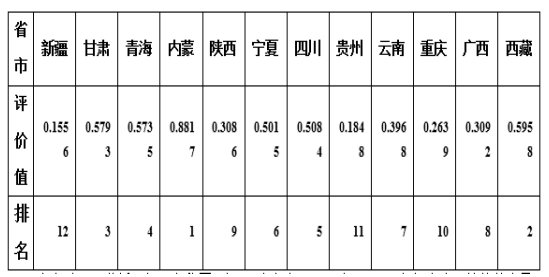

根据表 3-4 分析可知, 在我国西部 12 省 市中, 2012 年至 2017 年间生态环境整体上 最好的是内蒙、西藏、甘肃和青海; 四川、 宁夏、云南和广西的生态环境状况次之; 陕 西、重庆、贵州和新疆的综合评价最差。近 年来随着国家对西部生态发展的不断重视, 内蒙古的生态环境实现了大逆转。绿锁沙喉, 黄沙变绿洲, 这只是内蒙古生态实现大逆转 的一个缩影。而以前生态环境较好的贵州由 于近年来不断地经济发展与城镇化建设, 过 分注重经济的发展而不重视对污染的治理, 使得其在西部 12 省中的综合排名落在了后方。 这个整体综合排序与实际排序较吻合, 具有 一定的参考价值。

\section{4 结论}

本文依据生态环境的评价理论, 建立起 了生态环境的动态综合评价模型, 并对 2012-2017 年我国西部 12 个省市的生态环 境进行综合评价研究, 主要的研究结论包括 以下几方面:

（1）依据生态环境的相关评价理论体系, 结合我国西部 12 个省市的生态环境实际状 况, 建立了一套适合西部 12 省市生态环境 实际的评价指标体系, 主要包含资源、经济、 环境和社会等四个方面指标。
(2) 对建立好的指标体系采用拉开档次 法进行分析, 得到各个指标在不同时刻权重, 通过观察分析, 工业污染及其治理情况以及 造林面积等生态因素与人口的发展变化对西 部综合发展情况的影响最大。

(3) 最后, 本文将熵值法和理想点法等 多种方法综合起来对我国西部 12 省市的生 态环境进行了动态综合评价, 并将它们进行 了综合排序。从综合得分可知, 生态环境整 体上最好的是内蒙、西藏、甘肃和青海; 四 川、宁夏、云南和广西的生态环境状况次之; 陕西、重庆、贵州和新疆的综合评价最差。

\section{参考文献}

[1] Matthew A, Luck G Darrel jendrdtte the Urban Funnel Model and the Spatially Heterogeneous Ecological. Ecosystems, 2001,4(8):782-796.

[2] 张华.西部地区生态环境综合评价研究. 西北大学经济管理学院. 西南科技大学学 报.哲学社会科学版, 2008 .

[3] 赵国杰. 基于网络层次分析法的城市竞争 力评价指标体系研究.天津大学管理学院. 科学进步与对策, 2007 .

[4] 寿晖.基于 AHP一墑值法商业银行体系风 险指标预警研究. 北京工业大学 经济管 理学院.银行发展,2013.

[5] 曹连海,郝仕龙,陈南祥.农村生态环境指 标体系的构建与评价. 水土保持研 究,2010,17(05):238-240+244.

[6] 王金叶,程道品,胡新添, 李铭.广西生态环 境评价指标体系及模糊评价. 西北林学院 学报,2006(04):5-8.

[7] He Y. Assessment research of bijie drought risk based on cloud model. Journal of Risk Analysis and Crisis Response, 2013, 3(4): 192-200. 